\title{
Surgical Management of Cholesteatoma Growing Medially into the Petrous part of the Temporal Bone
}

\section{Maged B Naguib*}

ENT Department, University of Dammam, Dammam, Kingdom of Saudi Arabia

\begin{abstract}
In this article we describe the surgical management of petrous bone cholesteatoma, a rare form of an epidermoid cyst growing medially into the petrous part of the temporal bone. In our series of 5 patients, high resolution Computerized Tomography (CT) played an essential role in the diagnosis of this pathological entity. CT was also important in planning for the surgical access to this intricate part of the temporal bone. In all cases we performed a subtotal petrosectomy with skeletonization of the facial nerve bony canal to preserve the facial nerve function. We were able to remove totally the medially growing cholesteatoma with low morbidity.
\end{abstract}

\section{Introduction}

Petrous bone cholesteatoma describes an epidermoid cyst affecting the petrous portion of the temporal bone. It is a rare pathological entity with a reported incidence of 4 to $9 \%$ of all lesions affecting the petrous pyramid $[1,2]$. Petrous bone cholesteatoma is detected in about $3 \%$ of all patients diagnosed and treated for cholesteatoma in general [3]. Contrary to the more common middle ear cholesteatoma, petrous bone cholesteatoma grows medial to the otic capsule invading the inner ear and the facial nerve early before they are diagnosed.

Clinical presentation of petrous bone cholesteatoma is not only variable but may be misleading. It can present as a middle ear cholesteatoma, progressive or sudden facial nerve palsy, sensorineural hearing loss or a conductive hearing loss, secretary otitis media or intracranial complication.

Moreover, managing petrous bone cholesteatoma is a surgical challenge. The tendency for medial growth renders the surgical management of petrous bone cholesteatoma a difficult task owing to the central location of the otic capsule and the facial nerve as well as the proximity of vital intracranial structures namely the internal carotid artery, the jugular bulb, the lower cranial nerves and the brain itself. An even more difficult task is to be expected with further extension of the petrous bone cholesteatoma towards the clivus, the sphenoid sinus and the rhinopharynx [3].

In this study we report on the presentation and clinical picture of our series of patients having a petrous bone cholesteatoma. The management strategy adopted to deal with this difficult and rather uncommon otologic entity is also discussed.

\section{Materials and Methods}

This study is a retrospective review of the charts of five patients with petrous bone cholesteatoma presented to the ENT department, Suez Canal University, Ismailia, Egypt and University of Dammam, Dammam, KSA during the period from 2005 to 2010.

Charts were reviewed with particular attention to the presenting symptoms, history of the disease, condition of the facial nerve, clinical findings and audiometric results at presentation. All patients had a high resolution CT of the temporal bone for diagnosis and follow up (coronal and axial thin cuts in a bone window). Operative details were reviewed and noted with special attention to the surgical access, success in cholesteatoma exenteration and the surgical complications.

CT for follow up was done every 6 months in the first year and annually in the following years for up to 3 years to detect recurrence of cholesteatoma.

\section{Results}

Our series of patients with petrous bone cholesteatoma comprises 5 cases; 3 males and 2 females. Their ages ranged from 18 to 50 years with an average of 30 years. Petrous bone cholesteatoma affected the right ear in 3 cases and the left ear in the remaining 2. The duration of symptoms was short and generally did not exceed 2 years. The main symptom at presentation was a gradual hearing loss reported by all patients. Four cases presented with a sensorineural hearing loss which was severe to profound. One case presented with a moderate mixed hearing loss. An ear discharge was the second most common symptom which varied from a purulent discharge to an occasional bloody discharge. Facial nerve weakness was seen in $2(40 \%)$ of our patients at presentation; both were grade 3 on the House-Brackmann scale [4]. Vertigo and imbalance were not reported as a complaint in any of our cases. Four cases gave a history of a previous ear surgery on the same ear $(80 \%)$.

Based on the CT topographic location and extension and following the classification of Sanna et al. [5], our series of petrous bone cholesteatoma was classified into supralabyrinthine ( 2 cases), infralabyrinthine (2 cases), and infralabyrinthine apical (one case).

\section{Selected Cases}

\section{Case 1}

A 50-year-old man had a history of a gradual hearing loss in his right ear which started 3 years before presenting to our clinic and progressed to a total hearing loss at presentation. He denied any history of a balance disorder. On microscopic examination he had a normal looking left ear. His right ear showed a whitish mass in the attic region covered by a reddish polypoidal tympanic membrane. His facial nerve was intact on both sides. Audiometric evaluation showed no response in the right ear with normal hearing level in the left. CT scan showed a supralbyrinthine petrous bone cholesteatoma (Figure 1).

*Corresponding author: Maged B Naguib, ENT Department, University of Dammam, Dammam, Kingdom of Saudi Arabia, E-mail: magedbaher@yahoo.com

Received February 22, 2013; Accepted April 28, 2013; Published May 05, 2013

Citation: Naguib MB (2013) Surgical Management of Cholesteatoma Growing Medially into the Petrous part of the Temporal Bone. Surgery S12: 012. doi:10.4172/2161-1076.S12-012

Copyright: @ 2013 Naguib MB. This is an open-access article distributed under the terms of the Creative Commons Attribution License, which permits unrestricted use, distribution, and reproduction in any medium, provided the original author and source are credited. 


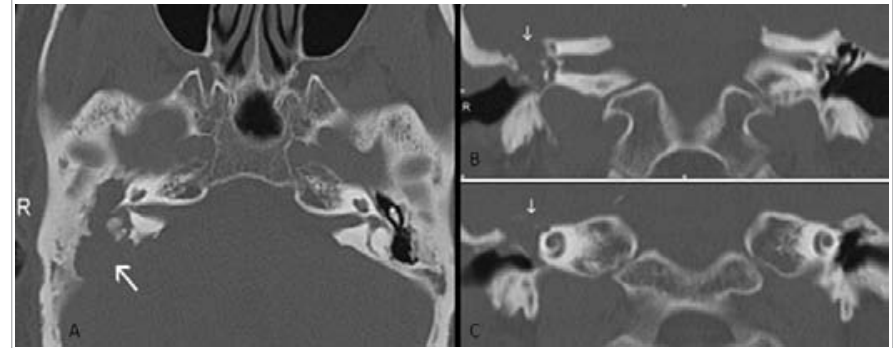

Figure 1: Right ear axial CT (A) showing a supralabytinthine petrous bone cholesteatoma eroding the inner ear vestibule and the bone of the posterio cranial fossa (arrow). Right ear coronal CT (B) showing erosion of the superior and lateral semicircular canals and the bone of the middle cranial fossa (arrow). Right ear coronal CT (C) showing erosion of the middle cranial fossa (arrow) but sparing the cochlea.

\section{Case 2}

A 24-year-old man presented with a right ear ache, discharge, hearing loss and a facial nerve weakness on the same side. He had a previous canal wall down mastoidectomy for removal of a middle ear cholesteatoma in the right ear at the age of 15 . At the age of 22 years, he underwent reconstruction of the right ear cavity using cartilage to reconstruct the outer attic mass with grafting of the tympanic membrane and obliteration of the mastoid cavity. He later on presented with a recurrent ear discharge and on examination the reconstructed attic was found perforated, with cholesteatoma extending medial to the cartilage used for reconstruction (Figures 2A and 2B). A follow up CT done after 8 months showed the development of an ifralabyrinthine petrous bone cholesteatoma (Figures 2C and 2D). Audiometric evaluation showed a moderate mixed hearing loss in the right ear and a mild conductive haring loss in the left ear.

\section{Case 3}

A 33-year-old woman came complaining of a hearing loss in the right ear and an occasional bloody ear discharge. She had undergone a canal wall down mastoidectomy in another hospital for removal of a right middle ear cholesteatoma two years before presenting to our clinic (Figure 3).

When seen, she had a grade 3 facial nerve paresis, total hearing loss and discharge with evidence of keratin in the right ear. Her left ear was normal. Audiometric evaluation showed a total hearing loss in the right ear with normal hearing in the left ear. CT scan showed an ifralabyrinthine apical petrous bone cholesteatoma.

\section{Discussion}

Our series of patients is small compared to the series being reported before. However, there are few authors worldwide reporting a series of more than 10 patients with petrous bone cholesteatoma.

Analysis of the information obtained from our small series of patients, one could come to some valuable conclusions. The small series in fact reflects the rarity of petrous cholesteatoma in our common practice. On the other hand, the small series can also reflect the lack of awareness of the general otolaryngologists as well as a number of otologists about petrous bone cholesteatoma invading medial to the otic capsule. We believe that both our conclusions are correct proved by the increase in number of detected cases in the recent couple of years especially among those who have had a previous ear surgery for the management of a middle ear cholesteatoma in the same ear. Therefore we agree with and highlight the recommendation of Sanna et al. [3], that a petrous bone cholesteatoma should be suspected in a patient with a history of an ear surgery for the management of cholesteatoma who develops an ear discharge and facial nerve palsy. In this case a high resolution $\mathrm{CT}$ is mandatory for the diagnosis.

Following this recommendation, we were able to detect, early, the transformation of the initial middle ear cholesteatoma in case (2) to a petrous bone cholesteatoma and to follow its medial growth into the inner ear.

Routine application of CT for the follow up of all patients with ear cholesteatoma in general has led to the fact that we did not encounter a single case of massive petrous bone cholesteatoma which involves the entire inner ear. Moreover, and contrary to the authors reporting an incidence of facial nerve paralysis in more than $50 \%$ of patients with petorus bone cholesteatoma $[3,6]$, we encountered this complication in only $40 \%$ of our cases owing to the early CT aided detection of the pathology. In case 3, the initial surgeon did not order for a preoperative $\mathrm{CT}$ as the patient was claustrophobic and depended only on the clinical examination showing evidence of keratin flakes. During surgery he discovered the medial growth of the cholesteatoma, terminated the surgery and followed the patient regularly, again without CT, till she developed a facial nerve weakness.
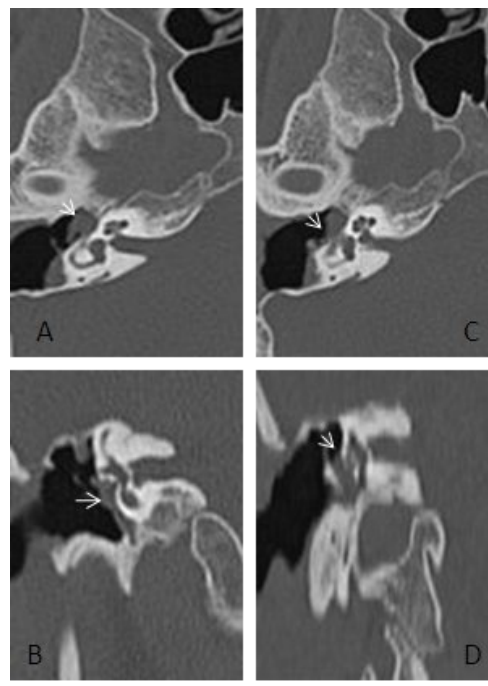

Figure 2: Initial axial CT of the right ear (A) showing a middle ear cholesteatoma (arrow). A coronal CT (B) shows the cholesteatoma (arrow) sparing the inner ear structures. A follow up axial CT (C) shows a medial invasion of the cholesteatoma into the inner ear (arrow). Coronal CT (D) shows destruction of the inner ear vestibule (arrow).
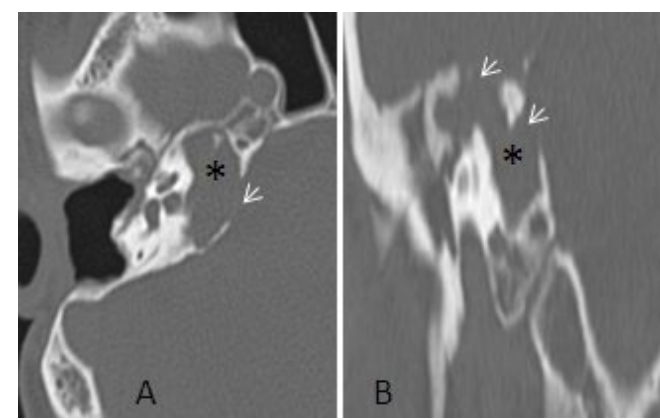

Figure 3: Right ear axial CT (A) showing the expansionof the petrous apex with a petrous bone cholesteatoma $\left({ }^{*}\right)$ with erosion of the posterior cranial fossa bony plate. Coronal CT (B) Showing cholesteatoma $\left(^{*}\right)$ and erosion of the cochlea and the posterior and middle cranial fossa dural plates (arrows). 
The value of a high resolution CT scan is not only in the detection of petrous bone cholesteatoma but in the exact localization of the lesion as well as in the determination of its route of spread. This is of a paramount importance in planning for the surgical access and the technique.

In our cases, supralabyrinthine cholesteatoma involved the anterior part of the epitympanum and extended medially and anteriorly to the petrous apex and the internal auditory canal. The infralabyrinthine type involved the hypotympanum below the inner ear capsule. The infralabyrinthine-apical type involved the hypotympanum and extended to the petrous apex and internal carotid artery anteriorly.

We surgically managed all our cases by performing a subtotal petrosectomy. This procedure entails a complete exenteration of all the pneumatized cells and tracts of the temporal bone with permanent obliteration of the Eustachian tube. The dura of the middle and posterior fossa is left covered by only a thin shell of cortical bone and the extent of otic capsule drilling is determined by the extension of the cholesteatoma itself. At times, the internal auditory canal had to be drilled and exposed to remove invading cholesteatoma as in case (3). At the end of the surgical procedure, the surgical cavity is obliterated with abdominal fat and the external canal is closed as a blind sac (Figure 4).

In order to preserve the integrity of the facial nerve function, we elected to leave the facial nerve in its bony canal as in the modified transotic approach [7]. The facial nerve canal is skeletonized by drilling the bone lateral and medial to the nerve. This leaves the facial nerve canal as a bridge across the surgical field with enough bone around it to protect it and to prevent accidental fracture while drilling (Figure 5).
In such a way, we could remove the medially growing petrous bone cholesteatoma completely.

Complications in our series were few. One of the cases with grade 3 facial nerve paralysis deteriorated to grade 6 one month after the surgical management. We had a single case of a postoperative cerebrospinal fluid leak which was managed by reopening the surgical cavity and obliterating it with more abdominal fat.

We are regularly following our patients with CT and MRI with fat suppression with no evidence of cholesteatoma recurrence.

\section{References}

1. de Souza CE, Sperling NM, da Costa SS, Yoon TH, Abdel Hamid M, et al. (1989) Congenital cholesteatomas of the cerebellopontine angle. Am J Oto 10: 358-363.

2. King TT, Benjamin JC, Morrison AW (1989) Epidermoid and cholesterol cysts in the apex of the petrous bone. Br J Neurosurg 3: 451-461.

3. Sanna M, Pandya Y, Mancini F, Sequino G, Piccirillo E (2011) Petrous bone cholesteatoma: classification, management and review of the literature. Audiol Neurootol 16: 124-136.

4. House JW, Brackmann DE (1985) Facial nerve grading system. Otolaryngo Head Neck Surg 93: 146-147.

5. Sanna M, Zini C, Gamoletti R, Frau N, Taibah AK, et al. (1993) Petrous bone cholesteatoma. Skull Base Surg 3: 201-213.

6. Moffat D, Jones S, Smith W (2008) Petrous temporal bone cholesteatoma: a new classification and long-term surgical outcomes. Skull Base 18: 107-115.

7. Gantz BJ, Fisch U (1983) Modified transotic approach to the cerebellopontile angle. Arch Otolaryngol 109: 252-256.

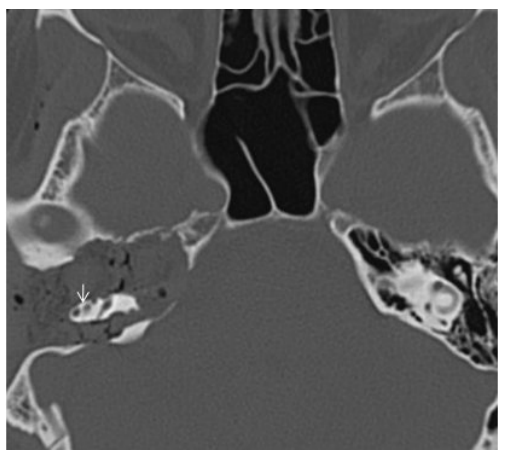

Figure 4: Right ear axial CT showing the postoperative cavity filled with abdominal fat. The facial nerve canal is shown in the middle of the surgical cavity (arrow).

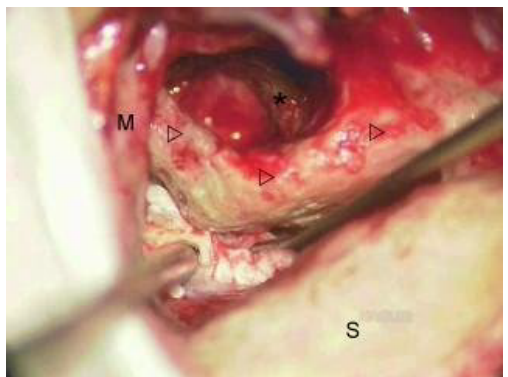

Figure 5: Intraoperative photograph of the right ear showing the dissection of the petrous bone cholesteatoma medial to the facial nerve.The dura of the middle cranial fossa (M), the sigmoid sinus (S), the facial nerve canal (arrow heads) and the internal carotid artery $\left({ }^{*}\right)$. 